\title{
A Fully individualized Adaptive and Intelligent Educational Hypermedia System: Details of CleverUniversity
}

\author{
Mohammed BOUSSAKUK ${ }^{1}$, Ahmed BOUCHBOUA ${ }^{2}$, Mohammed EL GHAZI ${ }^{3}$, Mohammed FATTAH ${ }^{4}$, \\ Moulhime EL BEKKALI ${ }^{5}$ \\ 1,2,3,5 Sidi Mohamed Ben Abdellah University, Fez, Morocco ${ }^{1}$ mohammed.boussakuk@usmba.ac.ma, \\ ${ }^{4}$ Moulay Ismail University, Meknes, Morocco, m.mohammedfattah@umi.ac.ma
}

\begin{abstract}
Traditional web-based learning platforms give the same educational content to each learner. Nowadays, these kinds of teaching environments are gradually replaced by individualized adaptive e-learning systems. This study deals with the design and implementation process of an innovative Dynamic Adaptive Hypermedia System (DAHS) named as "CleverUniversity". Learning styles are one of the most important factors in determining personal differences. Accordingly, our system allows students to take content of the course appropriate to their own needs and learning styles. By this means, the efficiency and effectiveness of learning activities would be improved. Our system is composed of five main components, first, learner's model, pedagogical model, domain model, content generator and multimedia database.
\end{abstract}

Key words : Adaptive assessment, Dynamic Adaptive Hypermedia, Individualized learning, Learning style.

\section{INTRODUCTION}

Today, learning environments and information and communication technology (ICT) are integral parts of an innovative education system. In traditional teaching methods offer predetermined courses in the same way to all learners[1]. Knowledge level, previous information, learning styles and individual characteristics and specification of students regarding the lesson are not taken into account in these environments. These differences may have a direct impact on the learning processes of learners. Dynamic Adaptive Hypermedia System (DAHS) is developed as an alternative to classical hypermedia systems according to "one-size-fits-all" approach by adapting to the needs and knowledge of each learner[2][3].

Design of DAHS is one of the new lines of research topics in the field of distance education. Key concept in these environments is learner modeling. In this study, an individualized adaptive e-learning framework based on learning styles (LS), CleverUniversity, was designed and developed. LS are typically defined as the behaviors and attitudes which determine the way students prefer to learn and train [4]. These students are different in terms of training, skills, attitudes, knowledge, motivations, needs and preferences. In order to improve the adaptability and individualization of learning in our system, we have chosen to adopt learning style models and a fine-grained approach to learning contents.

In the first part of this article, we describe the architecture and components of our System Adaptive Hypermedia Dynamic named as CleverUniversity. It is individualized based on the index of learning styles (ILS) developed by Felder and Silverman [5]. The system is developed using PHP5 language in Symfony Framework. MySQL was used for database. Section 2 deals integration an adaptive assessment module into CleverUniversity. Thanks to this module, learners receiving personalized assessment appropriate for their own ability level. Lastly, Section 3 deals with our conclusions and presentation of our future work.

\section{AN INNOVATIVE INTELLIGENT AND ADAPTIVE E-LEARNING SYSTEM: CLEVERUNIVERSITY}

This section deals with CleverUniversity architecture based on a client/server model. It was designed and encoded using Symfony PHP framework, JQuery, CSS and MySql database. CleverUniversity can be examined under seven main parts. These are architecture, learner module, domain module, pedagogical module, basis of multimedia resources, content generator user interface and adaptive testing module.

\subsection{Architecture of CleverUniversity}

Figure 1 below illustrates the basic architecture of our CleverUniversity environment. It was developed as an intelligent and adaptive e-learning platform where education content is generated for each learner according to his/her learner's model. Students and teachers should register to access to the system. The student who logs in the system takes the Felder-Silverman questionnaire. Hereby, learning style of this student is detected [4]. By this means, individualized education content will become more effective and adaptive[6]. 

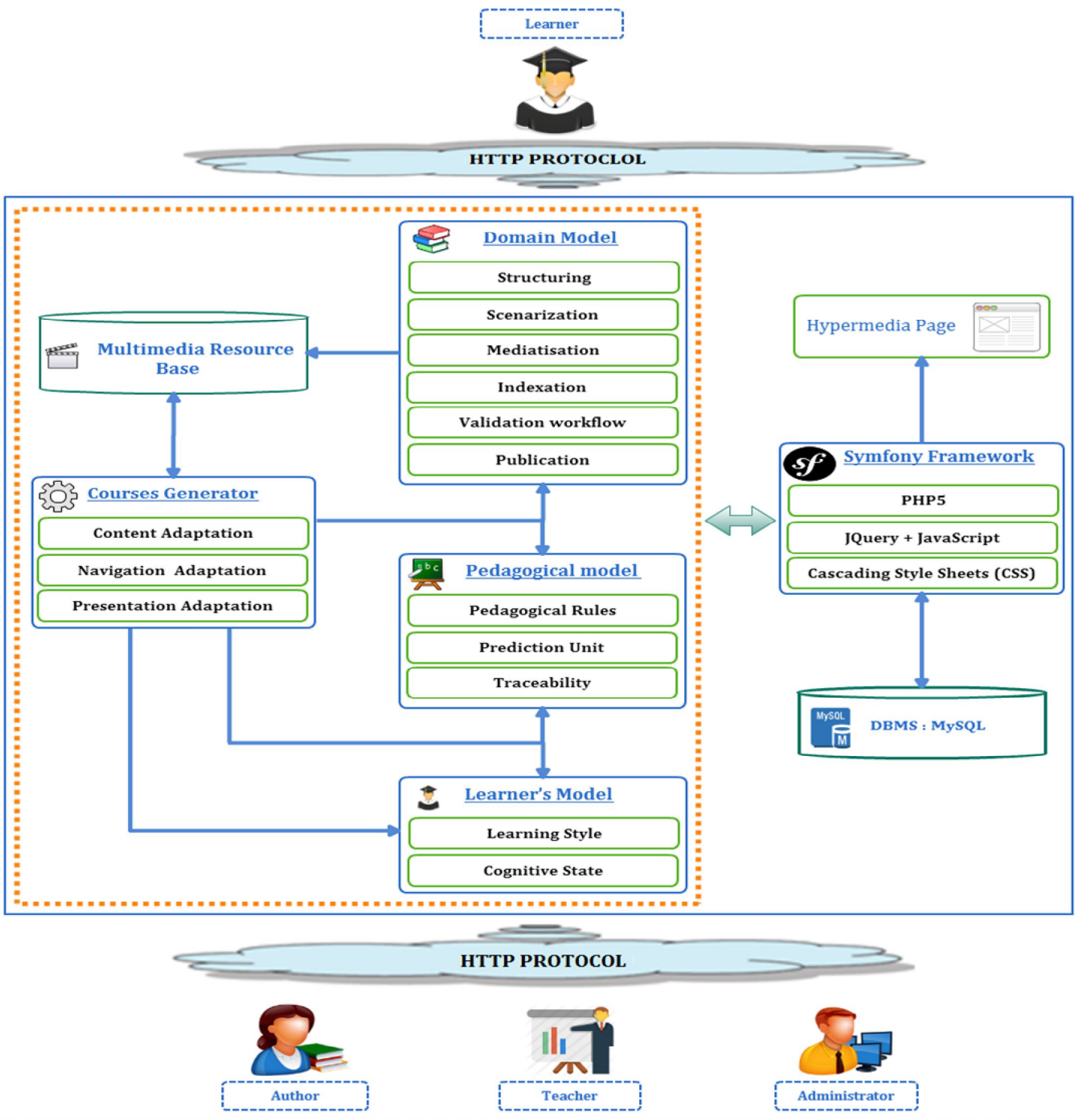

Figure 1: Architecture of CleverUniversity

\subsection{Learner module}

The intelligence of a Dynamic Adaptive Hypermedia System (DAHS) is primarily attributed to its capacity to offer personalized content according to the personal needs and abilities of each student. Our intention is to provide the learner with concepts tailored to her/his needs that permits to reduce the time spent in learning. Hence, this content should have a high educational quality. This can only be accomplished through knowledge of the student's model, which is one of the most important elements of our system. This module presents the kernel of any individualized learning and places the student in an interactive role in teaching process.

In addition, the information kept in learner module help to define the relevant characteristics of each learner that can better measure or describe his/her performance, motivation, cognitive level, goals, learning style and psychological problems. 
In our approach, every student must be modeled, firstly, by traits which are collected in a facet of identification with the personal data such as: username details, password, first and last name, day of birth, email, etc. Secondly by the goal and requirement of the student to the knowledge. The only way to acquire this information is through a questionnaire that the student must answer when he or she first accesses the platform. This questionnaire contains the ILS questions to predict his/her own learning styles.

Initial learning style of learner is calculated based on the learners' answers based on the learning style-model by Felder-Silverman. Consequently, learning style is recorded in appropriate learner model [7].

\subsection{Adaptation mechanism based on the student's learning profile}

We can summarize the process of adapting educational content based on the student's learning profile in six main steps:

Step 1: The first time that learners use CleverUniversity, system stores personal information supplied directly by the learner.

Step 2: The user is faced with two distinct decisions either: fill the questionnaire in Felder-Silverman and jump to the stage four; this consists of a set of psychological questions that easily identifies the student's habits, behavior and learning profile. Otherwise, the content builder proceeds to the step three.

Step 3: In the event that the student surpasses the questionnaire, a default learning profile that we have already defined will be assigned to the learner, and move to step five.

Step 4: All of the learner's responses to our survey will permit to the tool to identify levels of priority for each component of the F\&S model.

Step 5: It permits to measure the degree of adaptation of material to the level of preference.

Step 6: The student defines its goals and selects the lesson that he/she wishes to take, so that learning content will be presented according to his/her learning style and current state of knowledge.

\subsection{Domain module}

Domain module provides information on the essential learning material that will be taught. One of the main objectives of the domain module is to identify the pertinent topics and their connections and to give an overview design of the teaching. In this step, our system provides teachers with an intuitive tool to create relevant and structured educational content [5].

\subsection{Pedagogical module}

The main objective of DAHS is providing adequate assistance to the learner. In this regard, our pedagogical module detailed how to design the learning approaches used by instructors when delivering of the education material to students. It also includes the criteria used to define the learner's knowledge level. The pedagogical module is the set of requirements for the learning material to be produced by the platform as well as how and when it is to be produced. This component is based on a module of prediction to predict the next content which could be accessed by the student and also allows promoting his/her autonomy who has a need to have a guidance or to evaluate the effectiveness of the efforts he or she is making and those that remain to be made.

\subsection{Bank of multimedia resources}

This bank contains the concepts, learning material, tutorials and quiz to be delivered to the student, based on the knowledge model. These education contents are distinguished by attributes to which educational content should be delivered to the student. There are four kinds of attributes: the physical type, the cognitive level, the cognitive type and the learning goal.

\subsection{Content generator}

The module of content generator or course generator is considered the most important component in our system, because it connects the different modules mentioned above. This block comprises the process to follow to update the student's model according to his behavior and strategies for enhancing the adaptability of the e-Learning environment. Three kinds of adaptations can be distinguished:

- Content adaptation: is based on the generation of education content based on the learner's model.

- Navigation adaptation: enables adaptation of the scheduling of concepts that will be presented to the student.

- Presentation adaptation: the system modifies the layout and the visual appearance. This form of adaptation uses some preferences of the learner. For example, the system can hide some links if it estimates from the student model that such links take her to the irrelevant content.

\subsection{User Interface}

While developing CleverUniversity, we paid attention to implement flexible and user-friendly interfaces. Our system is based on a 3-tier architecture. A three-tier architecture is a client-server software architecture which is composed of 3 
layers of logical computing. This architecture provides many advantages for development systems by modularizing the user interface, business logic and data storage layers. CleverUniversity is based on Symphony which is a free, open-source PHP web framework, and MySQL was used for databases.

\section{ADAPTIVE ASSESSMENT MODULE: CLEVERTESTING}

At present, our system generates only traditional examinations (tests). However, this test format has many limitations and raises various problems of accuracy in measuring the learner's ability level. In order to solve these problems, we are working on the design and implementation of an innovative Computerized Adaptive Testing (CAT) module [8]. The primary distinguishing feature of CAT is that sets of items (questions) that are administered to examinees are specifically selected for each examinee during the evaluation process according to his/her cognitive abilities estimation [9].

We have designed and implemented an assessment authoring tool to assist teachers in their tasks of creating, presenting, scoring and storing evaluations and their results. Our tool named "CleverTesting" will allow the generation of questions and tests in accordance with the latest version of the IMSQuestion and Test Interoperability v2.2 specification (IMS-QTI) in order to ensure their interoperability and reusability between different compliant systems [10].

The QTI Specification describes a basic format for the representation of the smallest exchangeable unit called Item. It relates to the question data, its presentation materials, the response processing rules, the outcome variables and the associated feedback.

Figure 2 shows a simple choice question with one correct answer [11]. With the IMS-QTI v2.2 specification, the descriptive file for this item in XML format would be as shown in figure 3.From the example illustrated above, we can see that the code needed to describe a simple question is very complicated. This complexity and richness of the specification pushes us towards the development of tools for automatic tag generation that are smarter and more advanced than existing XML editors. In addition, these tools must assist the teacher-evaluator in his task of creating educational content, searching for it and importing it from other heterogeneous repositories.

In the second phase, in order to build a flexible platform for online CAT, we will need to apply the Item Response Theory (IRT), the most commonly used form of psychometric theories today. IRT is a "family of mathematical models that describe how people interact with test items" [8]. It is a statistical model that focuses on the design and analysis of test elements to establish the relationship between these evaluation content and examinees [12], to make the quiz generated by our tool more adaptive to the examinee's skill level.
Figure 4 shows the flow of the model on an adaptive assessment activity.

\section{UNATTENDED LUGGAGE}

Look at the text in the picture.

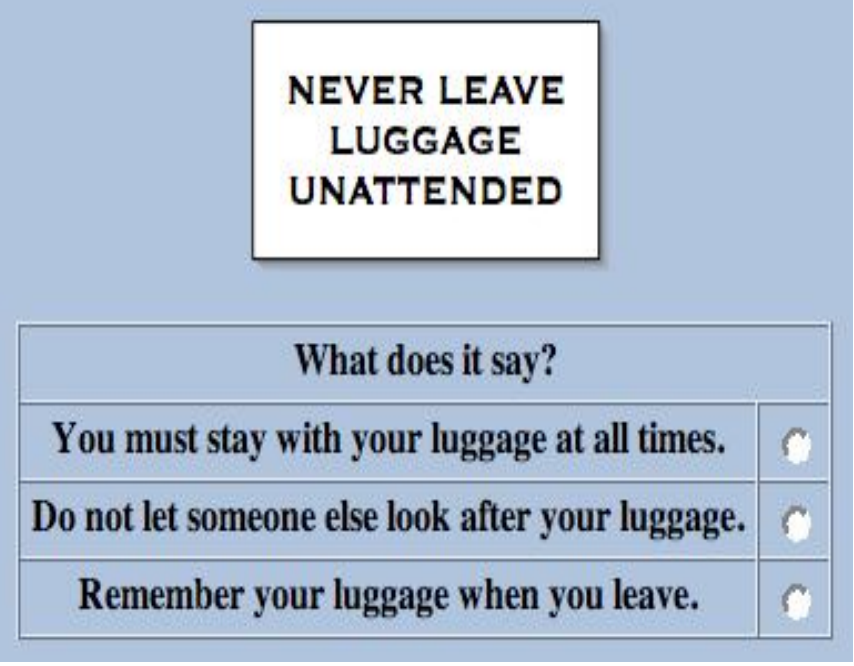

Figure 2: A simple choice question.

Finally, our CleverTesting plug-in will be integrated into our Dynamic Adaptive Hypermedia System (DAHS) called CleverUniversity, while considering its integration into other community projects such as Moodle. This phase will be followed by experiments to improve our system and evaluate its effectiveness.

\section{CONCLUSION AND FUTURE WORK}

In this paper, architecture and design of an Adaptive Hypermedia Dynamic System named CleverUniversity are focused on. CleverUniversity is a personalized adaptive e-learning environment. Our system identifies the learning styles of learners and presents the appropriate education content to each learner according to their own knowledge state and learning styles.

As future works, we are planning to develop the adaptive assessment module proposed in section 3 and its integration into our CleverUniversity. Thanks to this module, learners receiving individualized assessment according to their own competences and ability level. This is because, in contrast to traditional assessment, adaptive evaluation makes it possible to determine the cognitive level of items (questions) candidate can answer, and cognitive level of items they cannot answer. Briefly, our system offers what students need. 


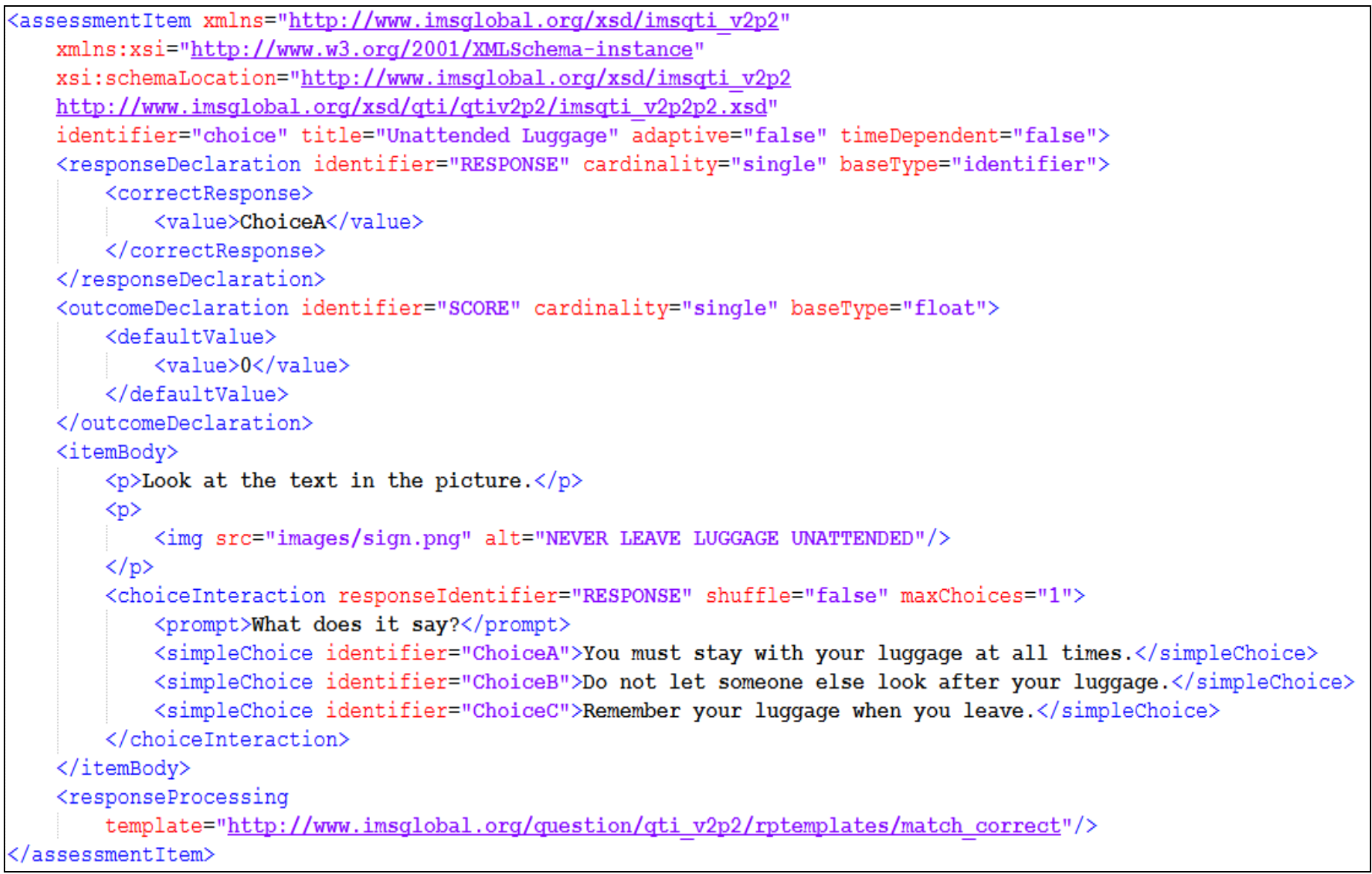

Figure 3: A single choice question coded in IMS-QTI version 2.2.

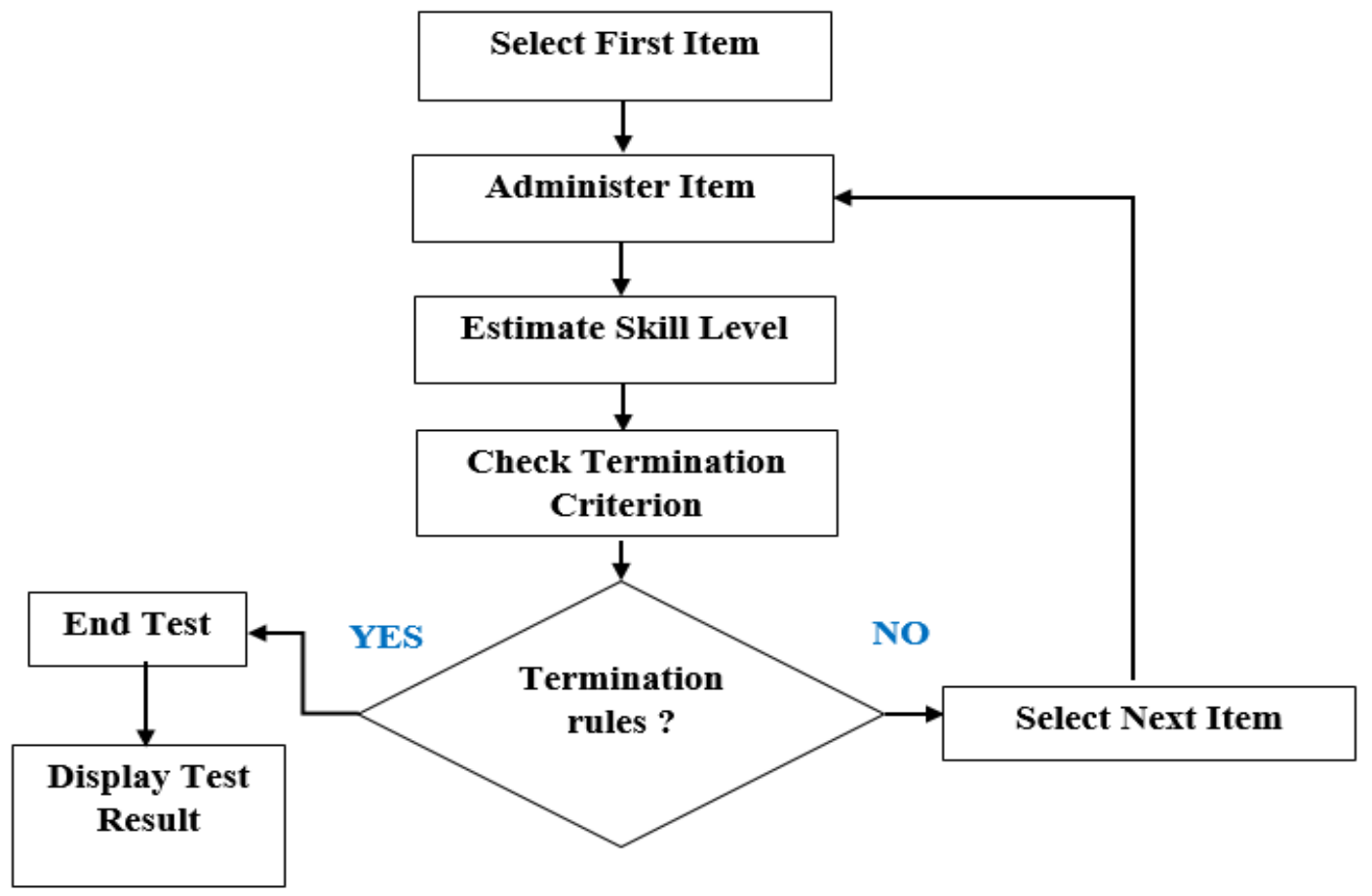

Figure 4 : Computerized Adaptive Testing Process. 


\section{REFERENCES}

[1] M. HALIM, N. ADADI, D. CHENOUNI, and M. BERRADA, "Web Services Composition in E-Learning platform," Int. J. Emerg. Trends Eng. Res., vol. 8, no. 2, pp. 525-532, 2020.

[2] A. Klašnja-Milićević, B. Vesin, M. Ivanović, Z. Budimac, and L. C. Jain, "Introduction to E-Learning Systems," in E-Learning Systems: Intelligent Techniques for Personalization, vol. 112, Cham: Springer International Publishing, 2017, pp. 3-17.

https://doi.org/10.1007/978-3-319-41163-7_1

[3] P. Brusilovsky, A. Kobsa, and W. Nejdl, The adaptive web: methods and strategies of web personalization. 2007.

https://doi.org/10.1007/978-3-540-72079-9

[4] J. Feldman, A. Monteserin, and A. Amandi, "Automatic detection of learning styles: state of the art," Artif. Intell. Rev., vol. 44, no. 2, pp. 157-186, 2015.

https://doi.org/10.1007/s10462-014-9422-6

[5] K.-M. Aleksandra, V. Boban, I. Mirjana, B. Zoran, and L. C. Jain, E-Learning Systems Intelligent Techniques for Personalization. Springer International Publishing, 2017.

[6] A. Bouchboua, R. Ouremchi, and M. Elghazi, "Educational Content Development Process in " CleverUniversity ": Our Dynamic Adaptive Hypermedia Environment," Int. J. Emerg. Technol. Learn., vol. 11, no. 12, pp. 14-22, 2016.

[7] A. Dhankhar and K. Solanki, "State of the Art of Learning Analytics in Higher Education," Int. J. Emerg. Trends Eng. Res., vol. 8, no. 3, pp. 868-877, 2020.

https://doi.org/10.30534/ijeter/2020/43832020

[8] S. Oppl, F. Reisinger, A. Eckmaier, and C. Helm, "A flexible online platform for computerized adaptive testing," Int. J. Educ. Technol. High. Educ., vol. 14, no. $1,2017$.

https://doi.org/10.1186/s41239-017-0039-0

[9] M. Boussakuk, M. El Ghazi, A. Bouchboua, and R. Ouremchi, "Online assessment system based on IMS-QTI specification," 2019 7th Mediterr. Congr. Telecommun., 2019.

https://doi.org/10.1109/CMT.2019.8931309

[10] "IMS Question and Test Interoperability (QTI): Assessment, Section and Item Information Model Version 2.2," 2015. [Online]. Available: http://www.imsglobal.org/question/qtiv2p2/QTIv2p2 -ASI-InformationModelv1p0/imsqtiv2p2_asi_v1p0_ InfoModelv1p0.html. [Accessed: 10-Jan-2020].

[11] "IMS Question \& Test Interoperability: Implementation Guide Version 2.2,” 2015. [Online]. Available:

http://www.imsglobal.org/question/qtiv2p2/imsqti_v 2p2_impl.html. [Accessed: 10-Jan-2020].
[12] Frank B. Baker, The Basics of Item Response Theory, Second edi. ERIC Clearinghouse on assessment and evaluation, 2001. 